Laser Chem. 1988, Vol. 8, pp. 335-348

(C) 1988 Harwood Academic Publishers GmbH

Photocopying permitted by license only

Reprints available directly from the Publisher

Printed in the United Kingdom

\title{
Cyclic Ether Auxofluors on Oligophenylene Laser Dyes
}

\author{
JOEL M. KAUFFMAN \\ Chemistry Department, Philadelphia College of Pharmacy and Science, \\ 43rd St. and Kingsessing Mall, Phila., PA 19104, USA
}

CHARLES J. KELLEY and ALEM GHIORGHIS

Chemistry Department, Massachusetts College of Pharmacy and A.H.S., 179 Longwood Ave., Boston, MA 02115, USA

EDWARD NEISTER and LORNE ARMSTRONG

Phase-R Corp., Old Bay Rd., New Durham, N.H. 03855, USA

(Received November 23, 1987)

Laser dyes with terphenyl and quaterphenyl as the fluorophors and with acyclic ether auxofluors were improved when the ether groups were made cyclic. Dyes containing cyclic ethers in 5- and 6-membered rings were observed to lase in the $360-400 \mathrm{~nm}$ region with better lifetime and/or solubility than their acyclic analogs. In one case the laser output wavelength of a quaterphenyl fitted with cyclic ether auxofluors was red-shifted enough to match the wavelength of a sexiphenyl without auxofluors.

KEY WORDS: Laser dyes, flashlamp-pumped, oligophenylenes, auxofluors, ethers.

\section{INTRODUCTION}

Oligophenylenes such as quaterphenyl 10 (Figure 4) have been among the most successful laser dyes in the ultraviolet region of the spectrum. ${ }^{1}$ The lack of solubility of most oligophenylenes, especially in polar non-toxic solvents, has severely limited their use. ${ }^{2}$ Attempts to place alkyl substituents on the internal ortho positions of the aromatic rings improves the solubility, but reduces both the fluorescence quan- 
tum yield (FQE) and the conversion efficiency. ${ }^{3,4}$ Both the quantum yield and the conversion efficiency can be restored, however, if ortho positions on adjacent rings are bridged by a single carbon substituted with gem-dialkyl groups. ${ }^{5}$ Additionally these bridged oligophenylenes show dramatically improved solubility properties in useful laser solvents. Placement of $t$-butyl groups on the outer meta positions of quaterphenyl gives a dye with fairly high output, but very short lifetime. ${ }^{4}$ Sulfonation of quaterphenyl produces a dye, "polyphenyl 1 ", which is soluble only in viscous solvents, and apparently suitable only for low power outputs. ${ }^{6}$ Attempts to promote solubility with alkoxy groups on the meta and para positions of the outer phenyl rings has little effect on solubility unless the groups are large ${ }^{2}$ as in the most successful ultraviolet-emitting dye in current use-BBQ, dye $\mathbf{1 0}$ (Figure 4). Even BBQ, however, is not soluble enough in methanol or ethanol for practical use in those solvents. ${ }^{5}$ Placement of alkoxy groups on either the outer meta positions or any internal ortho positions gives dyes with very short lifetimes. ${ }^{4}$

For some time we have sought a way to extend the useful range of the laser emission of oligophenylene laser dyes beyond the ultraviolet into the visible. Such a red-shift in the emission spectrum would require the attachment of auxofluors along the main axis of the oligophenylene to extend the conjugation of the $\pi$-electron system. Dialkylamino groups have been very successful auxofluors on coumarins, rhodamines, and other types of laser dyes. ${ }^{1}$ Cyclic dialkylamino groups, such as julolidine or $\mathrm{N}$-alkyltetrahydroquinolines incorporated into coumarins, rhodamines, and other types of laser dyes, give dyes with improved energy output and lifetime. ${ }^{7}$ The incorporation of a julolidine group also causes a red-shift in the laser output. However, Pavlopoulos $^{8}$ reported that simple dialkylamino substituents in para positions on terphenyl and quaterphenyl give unstable laser dyes. A terphenyl with a monocyclic dialkylamino group on one end, 6-(4biphenylyl)-1-ethyl-1,2,3,4-tetrahydroquinoline (dye 6, Figure 2, with $\mathrm{N}$-ethyl in place of $\mathrm{O}$ ), lased poorly when flashlamp pumped in toluene. ${ }^{9}$ Thus oligophenylenes substituted with alkylamino groups alone do not look promising as laser dyes, at least for the ultraviolet region.

A quaterphenyl substituted with methoxy groups in the para positions of the outer rings can be rendered soluble by the incorporation of $\mathrm{o}, \mathrm{o}^{\prime}$-bridging with a dipropylmethylene group. Dye 12 (Figure 5) 
bearing methoxy groups as auxofluors lases with no loss in lifetime vs. the corresponding dye lacking the ether auxofluors when flashlamp pumped. ${ }^{5}$ Additionally the laser emission of $\mathbf{1 2}$ is shifted to longer wavelength, and the energy output of the laser is enhanced when compared with that of the parent compound lacking the methoxy groups. One cyclic ether analog of $\mathbf{1 2}$ (dye 13 in Figure 5) has as high an energy output in ethanol as dye 12, and its emission spectrum is red-shifted by an additional $5 \mathrm{~nm}$. Since the properties of 13 have not been rationalized, ${ }^{5}$ they will be discussed in this paper as will our further efforts in the testing of cyclic ether auxofluors on oligophenylenes.

The incorporation of the more photochemically stable ether groups into alicyclic rings was expected to confer benefits similar to those of the rigidized amino groups, where the rigidized structure prevents collisional deactivation of the excited state of the molecule. ${ }^{10}$ Moreover, there is some evidence that rigidization of alkoxy groups on benzene rings makes their absorption of light more efficient, as shown by a $35 \%$ increase in the magnitude of the extinction coefficient on going from the acyclic ether 1 (Figure 1) to six-membered ether 3 , and
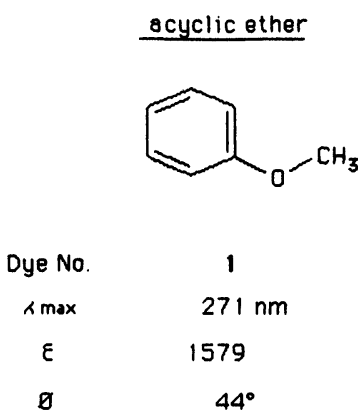
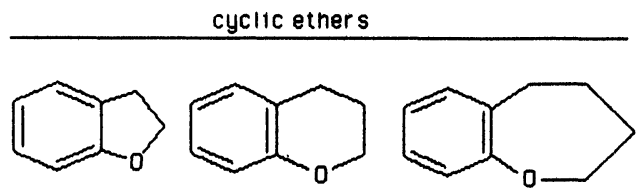

2
$283 \mathrm{~nm}$
3095
$0^{\circ}$

$0^{\circ}$

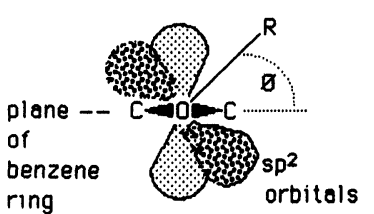

$\begin{array}{cc}3 & 4 \\ 276 \mathrm{~nm} & 267 \mathrm{~nm} \\ 2125 & 678 \\ 34^{\circ} & 62^{\circ}\end{array}$

maximum

interaction

when

D $=0^{\circ}$

Figure 1 Variation of light absorption maximum and extinction coefficient with $\theta$ in alkoxybenzenes (adapted from Reference 11). 
an increase of about $95 \%$ in going from 1 to the five-membered ether 2. ${ }^{11}$ The importance of high extinction coefficient ${ }^{12}$ for a laser dye may be seen in the relation:

Emission Intensity $=(2.3)($ Excitation Intensity $)($ Extinction Coefficient)(Optical Path Length)(FQE)(Fluor Conc.)

Clark et al. ${ }^{11}$ attributed the increase in extinction coefficient to enhanced conjugation of one of the non-bonded electron pairs on the other oxygen with the $\pi$-electrons of the benzene ring in the cyclic ethers. The angles $\theta$ of the $\mathrm{C}-\mathrm{O}-\mathrm{C}$ bonds of the ethers given in Figure 1 were calculated, where the completely planar $\left(\theta=0^{\circ}\right)$ five-membered ring of coumaran seems to represent the ideal case. Still smaller rings in which one would also expect $\theta=0^{\circ}$ would be thermally and/or photochemically unstable. ${ }^{29}$ The 7 -membered benzoxepine 4 had very poor conjugation as evidenced by its very low extinction coefficient.

Very high FQEs were reported by Berlman ${ }^{13,14}$ for a mono terminal alkoxyterphenyl related to dye $\mathbf{5}$, and for a dialkoxyterphenyl related to dye 8, and also for $3,3^{\prime \prime \prime}$-dimethylquaterphenyl, that is, for substitution in either the terminal para or terminal meta positions. Equally high FQE, high energy output as a laser dye, and good lifetime when $\mathrm{XeCl}^{*}$ laser pumped was reported for 3,3"' -dimethylquaterphenyl. ${ }^{4}$ Terminal methoxy groups on a quaterphenyl gave good lasing lifetime when flashlamp pumped, ${ }^{5}$ while the branched alkyl groups in dye $\mathbf{1 0}$ did not. Para-methoxyalkylarenes were very resistant to reaction with oxygen at $100^{\circ},{ }^{15}$ certainly a desirable feature in a laser dye. Joining these two types of substitution together, one can envision cyclic ethers of the type we now report upon.

\section{EXPERIMENTAL}

Dyes 1 and 4 were not handled in this work. Dye 2 is available from Aldrich Chemical Co. Dye 3 was first prepared by Deady's method, ${ }^{16}$ and later by a variant of Baddeley's method, ${ }^{17}$ which gave purer product. Dye 5 has been reported in poor yield from GombergBachmann coupling. ${ }^{15}$ We prepared dyes 5-7 by oxidative decarboxylation of the Diels-Alder adduct of dimethyl acetylenedicarboxylate 
and the appropriate diene as described for $p$-terphenyl by Fieser. ${ }^{18}$ The dienes were made by Wittig-Horner reactions. The aldehyde required for dye 5, $p$-anisaldehyde, is available from Aldrich. The aldehydes required for dyes 6 and 7 were prepared as described by Baddeley $e t$ $a l .{ }^{17}$ Dye 8 was prepared according to Ronlàn et al. ${ }^{19}$ The 6bromochroman required for dyes 9,11 and 13 was prepared according to Maitte $;^{20}$ a similar bromination gave the 5-bromocoumarans required for dye 14 and its lower homolog (see below); 5bromocoumaran has been reported. ${ }^{21}$ Dyes 9 and 11-14 were prepared by coupling the Grignard reagent of the bromoether with the appropriate dibromoarene in the manner of Pridgen and Jones. ${ }^{22}$ Dye 10 was obtained as a gift from Exciton Chemical Co. as "BBQ". The solvents DMA, DMF and cyclohexane were of spectrophotometric grade from Aldrich.

Solubilities of dyes at $20-23^{\circ}$ were determined either by shaking suspensions with incremental additions of solvent until the dye crystals disappeared, or by measuring the ultraviolet absorption of saturated solutions.

Ultraviolet spectra were determined with a Cary 15 spectrometer in $1 \mathrm{~cm}$ quartz cells at a concentration of $\approx 2.5 \times 10^{-5} \mathrm{M}$. The solutions used for determination of FQE were all adjusted to an absorbance of $0.1 \pm 10 \%$ using $10 \mathrm{~cm}$ quartz cells in the Cary 15 .

Fluorescence spectra were obtained with a Perkin-Elmer MPF-44A spectrometer (operated in ratio mode with Rhodamine B in the reference compartment) in conjunction with a Hitachi corrected spectra unit. Slit widths used were $1 \mathrm{~nm}$ for excitation and $3 \mathrm{~nm}$ for emission. FQEs were determined by the dilute solution method ${ }^{23}$ where the reference compound was $p$-terphenyl in cyclohexane, and its FQE was taken as $0.77 .{ }^{24}$ Areas of emission spectra were determined to $\pm 2 \%$ with a planimeter (Los Angeles Scientific Instrument Co. Model L20M).

The flashlamp-pumped laser was a Phase-R Model DL-1200. The volume of the dye solution was 0.3 liters, and it was pumped at 2-3 $1 . /$ min through a $0.3 \mu$ glass filter. The rise-time of the flashlamp was 25 ns. The output mirror had a reflectivity of $70 \%$ at wavelengths of $340-420 \mathrm{~nm}$. The energy input was determined by measuring the voltage. The energy output was found with a Laser Precision RK 3230 energy meter. 


\section{DISCUSSION AND RESULTS}

\section{4-Alkoxyterphenyls (Figure 2)}

There was no significant difference in the longwave absorption peak of dyes 5-7 in DMF. The extinction coefficient did not follow the pattern shown in Figure 1, although that of dye 7 was greater than that of dye 6. The single smooth fluorescence peak of each, however, followed the expected pattern, with the chroman 6 red-shifted $12 \mathrm{~nm}$ compared with acyclic 5 , and the coumaran 7 red-shifted $17 \mathrm{~nm}$ compared with 5 . The FQEs in DMF showed a value of 0.70 for dye 5 , in good agreement with 0.74 in cyclohexane reported by Berlman ${ }^{13,14}$ for the homolog 4-(3,3-dimethylbutoxy)-p-terphenyl. Dyes 6 and 7, surprisingly, had much lower FQEs. None of the three dyes lased when flashlamp pumped.

The melting points had the expected relative values. Construction of ball-and-stick molecular models shows that a terminal methoxy group does not protrude beyond the "line" of benzene rings, and packing in the crystal must be at least as good as that of $p$-terphenyl, MP $212^{\circ}$. Even a dihydrofuran ring is somewhat larger than a benzene ring, and

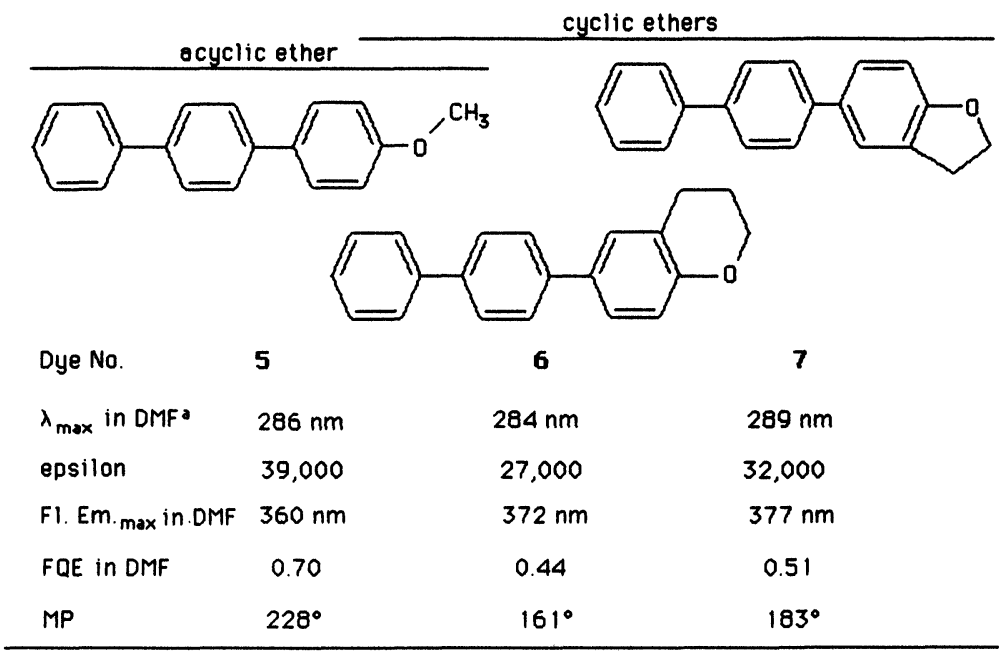

a N,N-dimethylf ormamide

Figure 2 Properties of 4-Alkoxyterphenyls. 
sticks out far enough to hinder packing. A dihydropyran ring, as in dye 6, is much larger than a benzene ring, and gives the lowest MP.

Despite the discouraging results, the red-shifts of fluorescence and effects on MP of the cyclic ethers showed that some of the properties expected were obtained. Even though dyes with similar ether auxofluors on both ends would not show much more red-shift, ${ }^{13,14}$ and the increased symmetry would not aid in lowering MP or increasing solubility, the slight improvements in FQE reported by Berlman for the symmetrical dialkoxy compounds gave us a reason to evaluate a pair.

\section{4,4"-Dialkoxyterphenyls (Figure 3)}

In dyes 8 and 9 , there was again no significant difference in the position of the longwave absorption peak; however, with this pair, at least the extinction coefficient was the same; a useful red-shift in fluorescence peaks was observed (these dyes have two peaks and a longwave shoulder in their fluorescence emission curves); and the FQE of the cyclic ether seemed a bit higher than for the acyclic ether. In this case, the cyclic ether 9 had much better lasing properties than the acyclic: (1) the lasing threshold was lower, and (2) the energy output was 3-5 times higher. (The commercial dye normally listed for this wavelength range, Butyl-PBD, barely lased under the same conditions.) Both peaked at $371 \mathrm{~nm}$ in DMA, and both had good lifetimes of about 30 $\mathrm{KJ} / 1$ in a system where Rhodamine $6 \mathrm{G}$ gave $45 \mathrm{KJ} / 1$.

Dye 9 had a lower MP than dye 8, and was actually somewhat more soluble in DMA. Dye 9 was soluble enough in ethanol for practical use, not so in methanol, but soluble enough in 1:9::DMA:methanol.

We did not attempt to prepare the coumaran version of dye 9 for two reasons: (1) the corresponding coumaranyl quaterphenyl (dye 14 without the outer four propyl groups) was not isolated during attempted synthesis by the usual method, probably due to oxidation of the furan ring, and (2) we had already made good dyes for the wavelength range expected $(\approx 380 \mathrm{~nm})$ with both better lasing properties, ${ }^{5}$ and most likely, better solubility.

\section{4,4"'-Dialkoxyquaterphenyls (Figure 4)}

The extension of the encouraging results with dye 9 to the next higher oligophenylene was attempted by synthesis of dye 11 and comparison 


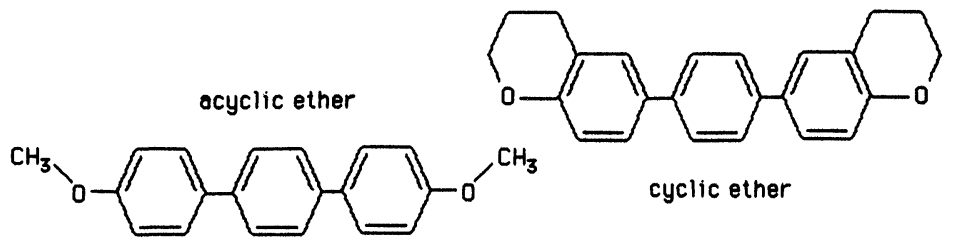

Dye No.

8

9

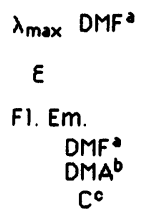

$288 \mathrm{~nm}$

$289 \mathrm{~nm}$

39,000

39,000

$359,348 \mathrm{~nm}$

356,340

$368,343 \mathrm{~nm}$
368,344

FQE DMF
DMA

DMA

0.57

0.65

0.69

0.63

0.69

\section{Lasing FPd}

Threshold $20 \mathrm{KeV}$

Energy Output in DMA $<1 \mathrm{mj}$

Peak in DMA

$371 \mathrm{~nm}$

Lifetimef

$\approx 30 \mathrm{KJ} / 1$

$16 \mathrm{KeV}$

$2 \mathrm{mj}$

$371 \mathrm{~nm}$

MP

\begin{tabular}{|c|}
\hline $\begin{array}{l}\text { Solubility } \\
\text { DMA } \\
\text { ethanol } \\
\text { methenol } \\
\text { 1:9::DMA:methanol }\end{array}$ \\
\hline $\begin{array}{l}\text { N,N-dimethylacetamide } \\
\text { cyclohexane } \\
\text { floshlomp pumped }\end{array}$ \\
\hline
\end{tabular}

$270^{\circ}$

$\approx 30 \mathrm{KJ} / 1$

$214^{\circ}$

$$
\begin{gathered}
\approx 10^{-3} \mathrm{M} \\
- \\
- \\
-
\end{gathered}
$$$$
\begin{aligned}
& 1.4 \times 10^{-3} M \\
& 1.2 \times 10^{-4} \\
& <10^{-4} \\
& 1.4 \times 10^{-4}
\end{aligned}
$$

Initiol pulse energy output of optimum input
Total pumping energy delivered to fleshlemp
per liter of lesing solution until loser output
energy is $50 \%$ of initiol volue

Figure 3 Properties of 4,4"-Dialkoxyterphenyls.

of it with BBQ (dye 10). The dimethoxy homolog of dye 10 is known, ${ }^{19}$ but the extreme drop in solubility ${ }^{2}$ going from terphenyls to quaterphenyls, often a factor of $\approx 50$, meant that the dimethoxy compound (dye 10 where $R=$ methyl) would not have been soluble enough for testing, while dye 10 itself is commercially available.

Here again, the absorption maxima, the extinction coefficients, the fluorescence emission peaks, and the FQEs in cyclohexane of dyes $\mathbf{1 0}$ 


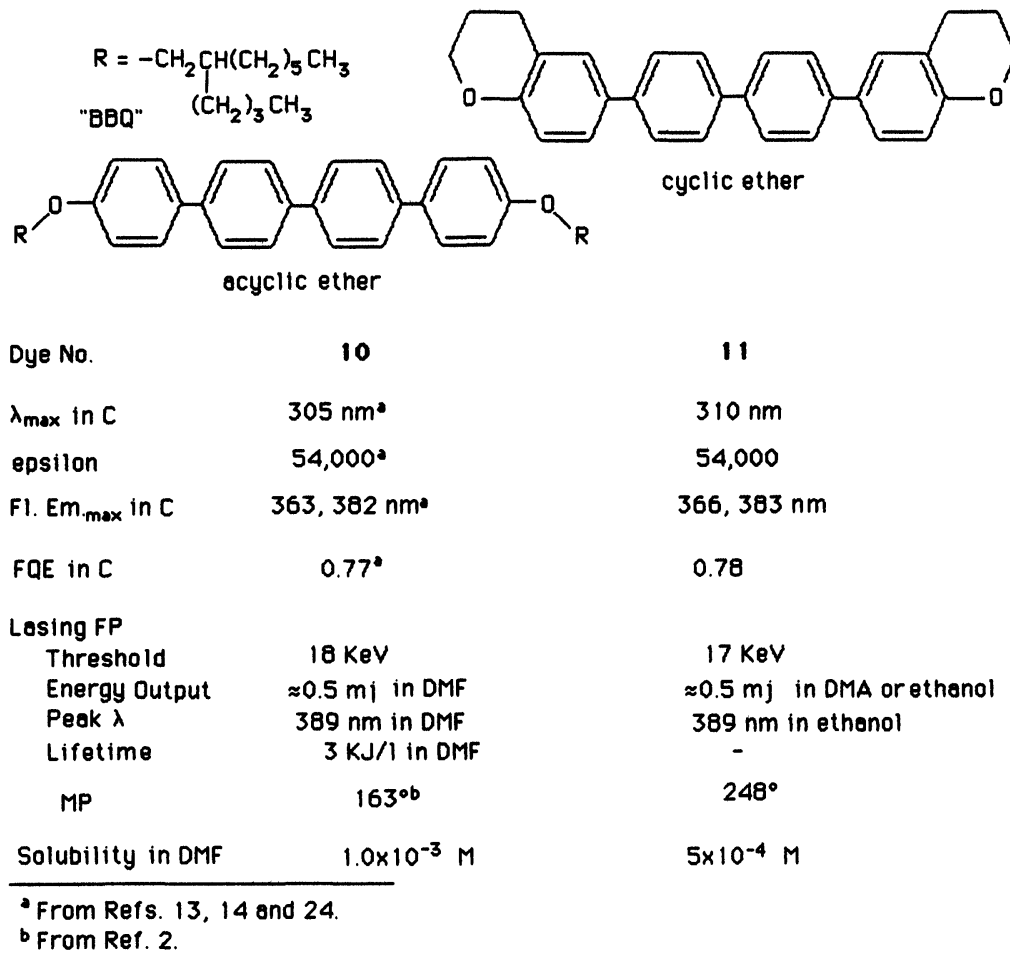

Figure 4 Properties of 4,4'"-Dialkoxyquaterphenyls.

and 11 were similar. Unlike the case with the dialkoxyterphenyls, however, the lasing performance was also similar, and not very good. One possible explanation for the difference is that accumulation of dye molecules in the $S_{1}$ state is necessary for lasing, so the lifetime of the $S_{1}$ state must need to be longer than what is necessary merely for a high FQE. It is well known that $S_{1}$ states of many types of aromatic dyes are planar with charge separation. ${ }^{10,25}$ Certainly any structural component that interferes with attainment of planarity of all aromatic rings reduces lasing output ${ }^{4,26}$ much more than it interferes with FQE. ${ }^{13,14}$ The most likely structure of the $S_{1}$ state of these symmetrical dyes is a planar one where one oxygen bears a substantial positive charge stabilized inductively by the other oxygen which bears as least a partial negative charge. ${ }^{25}$ There is actually an example from the field of 
electrochemistry which supports this finding. Under electrolysis conditions dialkoxyoligophenylenes form cation radicals by loss of one electron. More stable cation radicals form more easily and have longer lifetimes. In the series of para-substituted compounds $\mathrm{MeO}\left(\mathrm{C}_{6} \mathrm{H}_{4}\right)_{\mathrm{n}} \mathrm{OMe}$, maximum stability of ions was observed with $n=2$ and 3 , while the compound with $n=4$ gave ions more difficultly formed and less stable. In fact, for the $n=4$ compound (dye 10 where $R=$ methyl), the values for formation of both the cation radical and dication were approaching the low values for an isolated anisole group. ${ }^{19}$ More stable cations were actually obtained from an ethylene bridged 4,4'-dialkoxybiphenyl, and (most stable) a methylene bridged $4,4^{\prime}$-dialkoxybiphenyl. Such bridging of quaterphenyls has given laser dyes of very high energy output and low threshold. ${ }^{5,26}$ We have reported that dipropylmethylene bridged quaterphenyls make better laser dyes in all respects than their unbridged counterparts. ${ }^{5}$ The bridge enhances the interaction between the ends of the molecule, aiding charge dispersion. Therefore, all work on quaterphenyls with cyclic ether auxofluors described below involves compounds with dipropylmethylene bridging.

\section{4,4'"-Dialkoxy-3',2"-dipropylmethylenequaterphenyls (Figure 5)}

Dyes 12 and 13 were reported earlier, ${ }^{5}$ but additional data is given here. An attempt was made to prepare dye 14 without the outer four propyl groups; but no product was isolated. We think this was due to oxidation to the furan during Grignard coupling, which does, after all, involve oxidation of certain carbon atoms initially bearing bromine from an oxidation number of $1-$ up to $0 .{ }^{27}$ No Grignard coupling had been involved in the synthesis of the dihydrofuran (coumaran) dye 7. The resonance stabilization of furan lowers the energy for such an oxidation. The dihydropyrans (chromans) do not gain a comparable resonance stability on oxidation to pyrans. The well-known ${ }^{28}$ 2,2-dialkyl-2,3-dihydrobenzofurans are notoriously reactive to both acids $^{29}$ and radicals. So to prevent aromatization, as well as to minimize other types of reactivity, the less well-known ${ }^{30} 3,3$-dialkyl-2,3dihydrobenzofurans were chosen. To maximize solubility propyl groups were chosen over other simple alkyl groups. We found that in the series of 9,9-dialkylfluorenes from methyl to butyl, the dipropyl homolog had the lowest MP. While very large and/or branched alkyl 

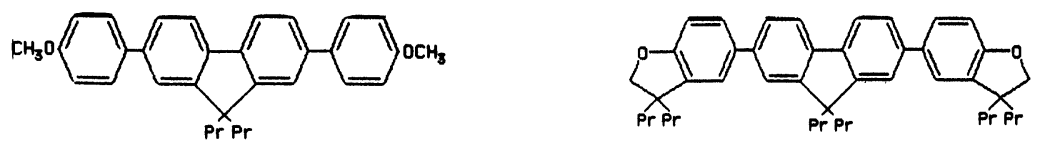

ocyclic ether

Dye No.

$\lambda_{\text {max }}$ in DMF

epsilon

FI. Em. $\lambda_{\text {max }}$

DMF

C

FQE

DMF

DMA

C

Losing FP in 1:4: :DMA:E

Threshold

Energy Dutput

Peak $\lambda$

Lifetime

MP

Solubility

DMA

ethanol

1:2::DMA:ethanol

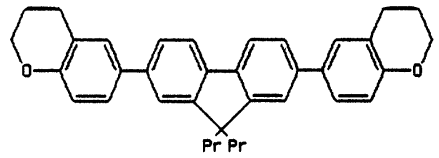

12

13

14

cyclic

ethers

$331 \mathrm{~nm}$
55,000
$371,389 \mathrm{~nm}$
372,390
366,384

$334 \mathrm{~nm}$

$335 \mathrm{~nm}$

52,500

52,300

$376,394 \mathrm{~nm}$

$-$

$380,399 \mathrm{~nm}$

374,392
0.90
0.73
0.78

0.89

0.73

$\begin{array}{ll}- & 0.73 \\ - & 0.82\end{array}$

$15 \mathrm{KeV}$
$12 \mathrm{~mJ}$
$386 \mathrm{~nm}$
$30 \mathrm{KJ} / 1$

$202^{\circ}$

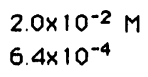

$15 \mathrm{KeV}$
$12 \mathrm{~mJ}$
$391 \mathrm{~nm}$
$30 \mathrm{KJ} / 1$

$213^{\circ}$
$5.0 \times 10^{-3} \mathrm{M}$
$3.4 \times 10^{-4}$

$14 \mathrm{KeV}$

$12 \mathrm{~mJ}$

$395 \mathrm{~nm}$

$45 \mathrm{KJ} / 1$

$188^{\circ}$

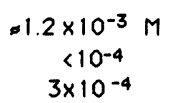

Figure 5 Properties of $4,4^{\prime \prime \prime}$-Dialkoxy-3',2"-dipropylmethylenequaterphenyls.

groups improve solubility of oligophenylenes in toluene, ${ }^{2}$ large alkyl groups are poor at increasing solubility in the desirable laser dye solvents DMF, DMA, ethanol, and methanol (compare the solubility of dyes 10 and 11 in DMF). Branched alkyl groups which have tertiary hydrogen atoms are more susceptible to attack by free radicals, ${ }^{29}$ and thus unstable under lasing conditions.

Dyes 12-14, as now seems "normal", showed small differences in the wavelengths of longwave absorption maxima, extinction coefficient, and FQE. The fluorescence emission spectra did show the 
expected progression, where the peaks of the chroman 13 were red-shifted $5 \mathrm{~nm}$ from those of acyclic dye 12, and the peaks of the coumaran 14 were red-shifted 8-9 $\mathrm{nm}$ from those of dye 12 in similar solvents. This means that dye $\mathbf{1 3}$ has emission at a wavelength longer than that expected from a quinquiphenyl without auxofluors, and dye 14 has emission at the wavelength expected for a sexiphenyl without auxofluors. All three dyes showed lower lasing threshold than their unbridged counterparts 10 and 11, and enough difference in output wavelength to be individually useful. The energy outputs of 12-14 were similar to each other and 5-10 times that of their unbridged counterparts. The lifetimes of dyes 12 and 13 were $\approx 30 \mathrm{KJ} / 1$, similar to dyes 8 and 9 . Dye 14 had the best lifetime: $\approx 45 \mathrm{KJ} / 1$, equal to that of Rhodamine $6 \mathrm{G}$ in this system, and much better than anything so far reported for flashlamp pumped UV dyes.

Dye 14 is soluble enough $\left(>2 \times 10^{-4} \mathrm{M}\right)$ in DMA for practical use, but not in ethanol, and probably not in methanol. A mixture of $1: 2::$ DMA:ethanol provides a suitable alternative solvent to neat DMA for dye 14. All the dyes are very soluble in dioxane.

Dyes $9,12,13$ and 14 are commercially available.

We are now investigating $4,4^{\prime \prime \prime \prime \prime}$-dialkoxysexiphenyls to provide laser dyes capable of peak output wavelength $>400 \mathrm{~nm}$.

\section{CONCLUSIONS}

Five new oligophenylene dyes $(6,7,9,11,14)$ containing cyclic ether auxofluors were prepared and their optical properties were compared with those of acyclic analogs and with those of one previously reported dye with cyclic ether auxofluors (13). The three new dyes with cyclic ether auxofluors at both ends lased as well or better than their acyclic counterparts. The dyes with an ether auxofluor at only one end did not lase at all, at least when flashlamp-pumped. The exact reason for the superiority of dialkoxyterphenyls 8 and 9 to the monoalkoxyterphenyls 5, 6 and 7 as laser dyes remains obscure.

In one case, the dye with cyclic ether auxofluors (9) showed lower threshold and much better energy output than its acyclic counterpart (8). In one case, better lifetime and more red-shifted lasing output was obtained from a dye with non-aromatizable dihydrofuran auxofluors (14) than from either a dihydropyran (13) or acyclic (12) counterpart. 


\section{Acknowledgments}

Discussions with James H. Bentley, US Army Missile Command, Redstone Arsenal, Huntsville, AL, USA; Aaron N. Fletcher, formerly at the Naval Weapons Center, China Lake, CA, USA; Theodore G. Pavlopoulos, Naval Ocean Systems Center, San Diego, CA, USA; and Richard N. Steppel, Exciton Chemical Co., Dayton, OH, USA, are greatly appreciated.

Computer-assisted literature searches by N. B. Rainey and technical assistance from S. J. Imbesi, L. Ruby, J. K. Adams and P. Erndwein are appreciated. Partial financial support for the work from the Philadelphia College of Pharmacy and Science Summer Faculty Research Fund is gratefully acknowledged.

\section{References}

1. M. Maeda, Laser Dyes: Properties of Organic Compounds for Dye Lasers (Academic Press, Tokyo, 1984).

2. H. O. Wirth, F. U. Herrmann, G. Herrmann and W. Kern, Molecular Crystals 4, 321 (1968).

3. W. Zapka and U. Brackmann, Appl. Phys. 20, 283 (1979).

4. M. Rinke, H. Güsten and H. J. Ache, J. Phys. Chem. 90, 2661 (1986).

5. J. M. Kauffman, C. J. Kelley, A. Ghiorghis, E. Neister, L. Armstrong and P. R. Prause, Laser Chem. 7, 343 (1987).

6. W. Hüffer, R. Schieder, H. Telle, R. Raue and W. Brinkwerth, Optics Comm. 33, 85 (1980).

7. K.-H. Drexhage in Topics in Applied Physics Vol. 1, Dye Lasers, F. P. Schäfer, Ed. (Springer-Verlag, Berlin, 1977).

8. T. G. Pavlopoulos and P. R. Hammond, J. Am. Chem. Soc. 96, 6568 (1974).

9. A. N. Fletcher, priv. commun., 1984.

10. G. Jones II, W. R. Jackson, C. Choi and W. R. Bergmark, J. Phys. Chem. 89, 294 (1985).

11. E. R. Clark and S. G. Williams, J. Chem. Soc. (B), 859 (1967).

12. S. G. Schulman, Molecular Luminescence Spectroscopy (Wiley, New York, 1985).

13. I. B. Berlman, H. O. Wirth and O. J. Steingraber, J. Phys. Chem. 75, 318 (1971).

14. I. B. Berlman, Handbook of Fluorescence Spectra of Aromatic Molecules, 2nd ed. (Academic Press, New York, 1971).

15. H. E. Mertwoy and H. Gisser, I\&EC Prod. Res. Dev. 6, 108 (1967).

16. L. W. Deady, R. D. Topsom and J. Vaughan, J. Chem. Soc. 2094 (1963).

17. G. Baddeley, N. H. P. Smith and M. A. Vickars, J. Chem. Soc. 2455 (1956).

18. L. F. Fieser and K. L. Williamson, Organic Experiments, 6th ed. (D. C. Heath, Lexington, MA, 1987).

19. A. Ronlán, J. Coleman, O. Hammerich and V. D. Parker, J. Am. Chem. Soc. 96, 845 (1974).

20. P. Maitte, Ann. Chim. (Paris) 9, 431 (1954).

21. C. K. Bradsher and D. C. Reames, J. Org. Chem. 46, 1384 (1981).

22. L. N. Pridgen and S. S. Jones, J. Org. Chem. 47, 1590 (1982).

23. J. N. Demas and G. A. Crosby, J. Phys. Chem. 75, 991 (1971).

24. J. B. Birks, Photophysics of Aromatic Molecules (Wiley-Interscience, London, 1970).

25. J. M. Kauffman and J. H. Bentley, Laser Chem. 8, 49 (1988).

26. M. Rinke, H. Güsten and H. J. Ache, J. Phys. Chem. 90, 2666 (1986).

27. J. M. Kauffman, J. Chem. Ed. 63, 474 (1986). 
28. C. D. Hurd and W. A. Hoffman, J. Org. Chem. 5, 212 (1940).

29. A. Streitwieser, Jr. and C. H. Heathcock, Introduction to Organic Chemistry (Macmillan, New York, 1976).

30. A. L. J. Beckwith and G. F. Meijs, J.C.S. Chem. Comm., 136 (1981). 